\title{
Results from background reduction in the DELPHI Forward RICH
}

\author{
J. Garcia-Perez et al
}

\begin{abstract}
The Forward Ring Imaging Cherenkov detector of DELPHI covers the forward region between $15^{\circ}<\theta<35^{\circ}$ and $145^{\circ}<\theta<165^{\circ}$. The full detector was operated during the 1994 LEP run period. The background reduction algorithms applied to the 1994 data are presented. The observed Cherenkov angle distributions for tracks with saturated angles are used to evaluate the performance of the algorithms. Results are given for dimuon and hadronic events.
\end{abstract}

Contribution to RICH'95, International Workshop on RICH Counters, Uppsala 12-16 June 1995 



\title{
Results from Background Reduction in the DELPHI Forward RICH
}

\author{
J. García Pérez a * and A. López Agüera b \\ a Universidad de Cantabria, Av. de los Castros, E-39005, Santander, Spain

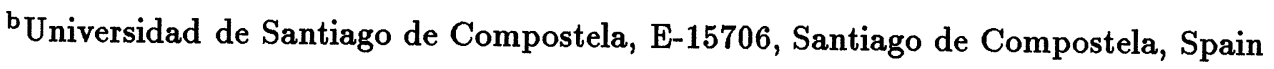

The Forward Ring Imaging Cherenkov detector of DELPHI covers the forward region between $15^{\circ}<\theta<35^{\circ}$ and $145^{\circ}<\theta<165^{\circ}$. The full detector was operated during the $1994 \mathrm{LEP}$ run period. The background reduction algorithms applied to the 1994 data are presented. The observed Cherenkov angle distributions for tracks with saturated angles are used to evaluate the performance of the algorithms. Results are given for dimuon and hadronic events.

\section{Introduction}

The Forward Ring Imaging Cherenkov (Forward RICH) detector [1] is part of the particle identification system of the DELPHI experiment [2] at the LEP collider at CERN. It is used to do hadron identification over most of the momentum range up to $40 \mathrm{GeV} / \mathrm{c}$ by Cherenkov angle reconstruction. In the first part of this paper a short description of the detector will be given. In a second part, the performance during the 94 running will be addressed. Using both, dimuon $(Z \rightarrow \mu \mu)$ and hadronic ( $Z \rightarrow$ hadrons) event samples on which a track selection has been applied, different algorithms to improve the signal-background ratio of reconstructed photoelectrons will be tested.

\section{Description of the Detector}

The Forward RICH detector covers the forward-backward regions $\left(15^{\circ}<\theta<35^{\circ}\right.$ and $\left.145^{\circ}<\theta<165^{\circ}\right)$. In azimuth the detector is segmented into sectors of $30^{\circ}$. Two radiator systems are used to cover the momentum range, a liquid $\mathrm{C}_{6} \mathrm{~F}_{14}$ layer for hadrons up to a momentum of about $6 \mathrm{GeV} / \mathrm{c}$ and a gaseous $\mathrm{C}_{4} \mathrm{~F}_{10}$ radiator volume for hadrons at high momentum. The imaging of the Cherenkov light from the liquid radiator is done by proximity focusing. Light from the gas radiator is focused by spherical mirrors onto a photo-sensitive Time Projection Chamber (TPC), read out by MWPCs with a gain around $2 \cdot 10^{5}$. Both anode wires and cathode strips

\footnotetext{
*Corresponding author
}

are read out and space-points are reconstructed from time matching of corresponding wires and strips hits. The analog electronics must be able to detect very small signals from single photoelectrons, while not being disturbed too much by the large signals from ionizing tracks.

\section{Background sources}

Apart from the signals coming from the Cherenkov light, there are other sources of hits which constitute a background. The following will be discussed:

- Feedback photoelectrons. They are created when U.V. light emitted during the gas amplification process in the MWPC's convert to electrons in the presence of the photosensitive vapour (TMAE). Optical screens are designed to minimize this effect, or at least confine it to a single wire. However sometimes feedback photoelectrons drift to neighbour wires.

- Cross-talk. Large signals, mainly from ionizing tracks, induce signals in neighbour strips of the same cathode block, and occasionally on neighbour wires.

- Particle showers crossing the detector. When many ionizing tracks traverse the detector, a number of ghost wire-strip associations are created. These events originate mainly from electromagnetic showers. 


\section{Background tagging algorithms}

Two kinds of algorithms have been identified $[3],[4]$ to tag the background. The first is based on the search for high hit densities produced by local phenomena like the crossing of a track. In the second group we use the tracks reconstructed by DELPHI to tag hits in their neighbourhood. Some algorithms are applied on hits (wires or strips) and others on space-points.

- Neighbours. If there are more than 3 space-points in the volume defined by a sphere of radius $1 \mathrm{~cm}$ around every spacepoint, all the space-points inside this volume are flagged.

- Track. If more than 5 adjacent wires or strips are fired with a maximum time difference between wires of $110 \mathrm{~ns}$, or between strips $90 \mathrm{~ns}$, then they are flagged as coming from a track.

- Cylinder. A cylindrical volume is defined to have the axis along the track trajectory reconstructed in DELPHI and a radius of 1 cm. All space-points inside are flagged as belonging to the track.

- $R \phi$ clustering. This algorithm deals with the hit density around the track. Planes perpendicular to the reconstructed track are divided in cells along the radial distance from the track and the azimuthal angle. An estimate of the cell hit density is made from the expected azimuthal isotropy of the Cherenkov photons and the background level. This algorithm can be tuned to optimize the background rejection power. If the density in the cell is bigger than the estimated one, all hits into the cell are flagged.

- Cluster multiplicity. Is defined as the number of times that a wire signal is used to build up a space point, matching with different strip clusters. We set a limit to improve the signal/noise ratio.
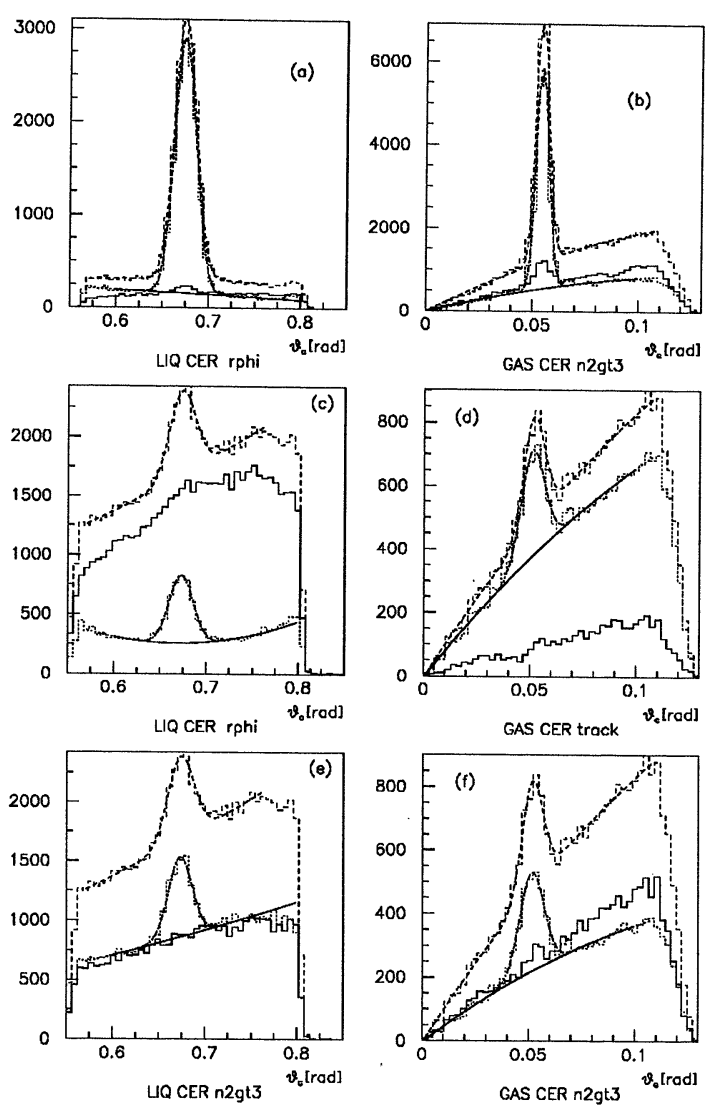

Figure 1. Cherenkov angle distributions for single photons (dashed line is without cut, solid what is catted and dotted what remains after cut). Dimuon events in the liquid with $R \phi$ cut (a),in the gas with neighbour cut (b), and for hadronic events in the liquid with $R \phi$ cut (c) and neighbour cut (e) and gas with track cut (d) and neighbour cut (f). 


\section{Definition of sample and signal}

\subsection{Samples used}

We have used two samples for the analysis, a dimuon sample from data taken in the 1994 running period and a full data sample (mainly hadronic events). The track selection was based on track momentum and quality. Both the main tracking detector, TPC and the chamber behind the Forward RICH, FCB, were required in the track. These criteria reduce the error on the position as well as on the momentum reconstruction. In order to get saturated Cherenkov rings we used tracks between 35 and $50 \mathrm{GeV} / \mathrm{c}$ in dimuon events. For hadronics, we require in the liquid radiator $5 \mathrm{GeV} / \mathrm{c}<P<30 \mathrm{GeV} / \mathrm{c}$, and in the gas radiator $5 \mathrm{GeV} / \mathrm{c}<P<9 \mathrm{GeV} / \mathrm{c}$.

\subsection{Fitting signal and background}

The distribution of Cherenkov angles of the photoelectrons is fitted to a gaussian plus a $2^{\text {nd }}$ order polynomia. The signal is defined as the area inside the gaussian between $\pm 3 \sigma$ around the mean value, and the area under the polynomia between the same limits is the background.

We define $N_{b}^{f}$ and $N_{b}^{i}$ as the number of photoelectrons from background after and before the cut, respectively, and $N_{s}^{f}$ and $N_{s}^{i}$ as the number of photoelectrons from signal after and before the cut respectively.

The background rejected is defined as $R=1-\left(N_{b}^{f} / N_{b}^{i}\right)$. The signal lost is defined as $N_{s}^{f} / N_{s}^{i}$. The signal over background ratio is defined as $N_{s}^{f} / N_{b}^{f}$.

\section{Results}

Tables 1-5 show the results. One can see that the application of the algorithms is quite effective. For the dimuon sample, the events have low background. Nevertheless, in the liquid, $R \phi$ still removes $\approx 50 \%$ of background with a minimum cut on signal. For the gas, the most effective is the neighbours cut $(n 2>3)$ which again removes more than $50 \%$ of background while cutting $\approx 10$ $\%$ of the signal. The track algorithm is more effective in the gas as the rings are smaller and sit very near to the track. What is more interesting for physics are hadronic events. The errors here are bigger due to larger track reconstruction errors and contamination of other particles than pions in the sample. We have much more background (s/n ratio below 1). We see from the correlation table that $R \phi$ is quite correlated with the other cuts. Also, neighbours is correlated with mult $>1$. In the liquid, $R \phi$ removes more than $80 \%$ background. In the gas, practically no cut removes signal. Again, most powerful is the neighbours cut, although the track cut is quite important, too.

\section{Conclusions}

Good rejection factors are obtained with a set of cleaning algorithms applied on a hadronics sample. They allow us to pass from a $\mathbf{s} / \mathbf{n}$ ratio of 0.2 to $\mathbf{1 . 0}$ for the liquid radiator. In the gas we go from 0.3 to 0.6 . This facilitate the extraction of the signal in a high background environment.

\section{Acknowledgments}

We thank all collaborators of the RICH collaboration for the fruitful discussions and encouragement during the work. The work has been supported by CICYT under contract AEN-0832 and by HCM program of EEC under contract ERBCHRXCT930345.

\section{REFERENCES}

1 W.Adam et al., The Forward Ring Imaging Cherenkov Detector of DELPHI Nucl. Instr. and Meth. A343 (1994) 284.

2 P. Aarnio et al., The DELPHI Detector at LEP, Nucl. Instr. and Meth., A303(1991)233276.

3 D. Bloch, M. Dracos, S. Tzamarias, Noise Simulation and Rejection for the DELPHI Barrel Ring Imaging Cherenkov Detector, presented at this workshop.

4 J. García, A. López, Background Reduction in the Forward RICH Detector of DELPHI, Nucl. Instr. and Meth., A343(1994)276-278. 
Table 1

Percent of Signal lost, Background rejection power, signal/noise ratio and number of signal photons for various noise rejection algorithms in the liquid radiator, for the Dimuon events. ORED means OR of neighbour, track and $R \phi$.

\begin{tabular}{llccc}
\hline Cut & sig. lost(\%) & Rej. (\%) & s/n Ratio & Nb sig ph \\
\hline neighbour & $2.3 \pm 1.0$ & $33.5 \pm 1.0$ & $5.6 \pm 0.1$ & 7.3 \\
track & $0.3 \pm 1.0$ & $9.1 \pm 1.0$ & $4.4 \pm 0.1$ & 7.4 \\
$R \phi$ & $2.9 \pm 1.0$ & $47.7 \pm 1.0$ & $8.0 \pm 0.2$ & 7.2 \\
mult $>1$ & $7.9 \pm 1.0$ & $32.8 \pm 1.0$ & $5.7 \pm 0.1$ & 6.8 \\
ORED & $4.8 \pm 1.0$ & $50.5 \pm 1.0$ & $7.9 \pm 0.2$ & 7.1 \\
\hline
\end{tabular}

Table 2

Same as Table 1 in the gas radiator for the Dimuon events. ORED means OR of neighbour, track and cylinder.

\begin{tabular}{llccc}
\hline Cut & sig. lost(\%) & Rej. (\%) & s/n Ratio & Nb sig ph \\
\hline neighbour & $10.4 \pm 0.9$ & $55.0 \pm 0.9$ & $4.1 \pm 0.08$ & 7.4 \\
track & $1.1 \pm 0.9$ & $23.5 \pm 0.9$ & $2.6 \pm 0.05$ & 8.1 \\
cylinder & $0.0 \pm 0.9$ & $17.6 \pm 1.0$ & $2.5 \pm 0.05$ & 8.3 \\
ORED & $14.7 \pm 0.9$ & $57.2 \pm 1.0$ & $4.1 \pm 0.08$ & 7.0 \\
\hline
\end{tabular}

Table 3

Same as Table 1 in the liquid radiator for the Hadronic events. ORED means OR of neighbour, track and $R \phi$ and mult $>1$.

\begin{tabular}{llccc}
\hline Cut & sig. lost(\%) & Rej. (\%) & s/n Ratio & Nb sig ph \\
\hline neighbour & $14.0 \pm 3.8$ & $48.7 \pm 3.8$ & $0.3 \pm 0.02$ & 5.8 \\
track & $0.8 \pm 4.9$ & $14.5 \pm 4.9$ & $0.2 \pm 0.02$ & 6.7 \\
$R \phi$ & $25.1 \pm 2.9$ & $81.0 \pm 2.9$ & $0.9 \pm 0.05$ & 5.0 \\
mult $>1$ & $13.4 \pm 4.4$ & $40.8 \pm 4.4$ & $0.3 \pm 0.02$ & 5.8 \\
ORED & $36.3 \pm 3.1$ & $85.6 \pm 3.1$ & $1.0 \pm 0.05$ & 4.3 \\
\hline
\end{tabular}

Table 4

Same as Table 1 in the gas radiator for the Hadronic events.ORED means OR of neighbour, track and cylinder and mult $>2$.

\begin{tabular}{llccc}
\hline Cut & sig. lost(\%) & Rej. (\%) & s/n Ratio & Nb sig ph \\
\hline neighbour & $0.1 \pm 4.8$ & $54.7 \pm 4.8$ & $0.6 \pm 0.06$ & 4.4 \\
track & $0.1 \pm 5.7$ & $19.9 \pm 5.7$ & $0.4 \pm 0.04$ & 4.6 \\
cylinder & $0.1 \pm 6.1$ & $5.9 \pm 6.1$ & $0.3 \pm 0.04$ & 4.2 \\
mult $>2$ & $0.1 \pm 6.0$ & $12.3 \pm 6.0$ & $0.3 \pm 0.03$ & 4.2 \\
ORED & $3.5 \pm 5.0$ & $59.7 \pm 5.0$ & $0.6 \pm 0.06$ & 4.0 \\
\hline
\end{tabular}

Table 5

Correlation between the various algorithms in the liquid radiator for the Hadronic events.

\begin{tabular}{llccc}
\hline$(\%)$ & neighbour & trk & $R \phi$ & mult $>1$ \\
\hline neighbour & 100 & 29 & 57 & 45 \\
track & & 100 & 17 & 20 \\
$R \phi$ & & 100 & 44 \\
mult $>1$ & & & & 100 \\
\hline
\end{tabular}

